\title{
Redox process is crucial for inhibitory properties of aurintricarboxylic acid against activity of YopH: virulence factor of Yersinia pestis
}

\author{
Alicja Kuban-Jankowska ${ }^{1}$, Kamlesh K Sahu ${ }^{2,3, *}$, Pawel Niedzialkowski ${ }^{4,}{ }^{,}$, Magdalena \\ Gorska ${ }^{1}$, Jack A Tuszynski ${ }^{2,3}$, Tadeusz Ossowski ${ }^{4}$, Michal Wozniak ${ }^{1}$ \\ ${ }^{1}$ Department of Medical Chemistry, Medical University of Gdansk, Gdansk, Poland \\ ${ }^{2}$ Department of Physics, University of Alberta, Edmonton, Canada \\ ${ }^{3}$ Li Ka Shing Institute of Virology, University of Alberta, Edmonton, Canada \\ ${ }^{4}$ Department of Analytical Chemistry, Faculty of Chemistry, University of Gdansk, Gdansk, Poland \\ *These authors have contributed equally to this work \\ Correspondence to: \\ Kuban-Jankowska Alicja, e-mail: alicjakuban@gumed.edu.pl \\ Keywords: Pathology Section, ATA (aurintricarboxylic acid), YopH, protein tyrosine phosphatase inhibitor, virulence factor \\ inhibition, oxygen reduction/oxidation \\ Received: May 25, 2015 \\ Accepted: July 09, 2015 \\ Published: July 22, 2015
}

\section{ABSTRACT}

YopH is a bacterial protein tyrosine phosphatase, which is essential for the viability and pathogenic virulence of the plague-causing Yersinia sp. bacteria. Inactivation of YopH activity would lead to the loss of bacterial pathogenicity. We have studied the inhibitory properties of aurintricarboxylic acid (ATA) against YopH phosphatase and found that at nanomolar concentrations ATA reversibly decreases the activity of YopH. Computational docking studies indicated that in all binding poses ATA binds in the YopH active site. Molecular dynamics simulations showed that in the predicted binding pose, ATA binds to the essential Cys403 and Arg409 residues in the active site and has a stronger binding affinity than the natural substrate (pTyr). The cyclic voltammetry experiments suggest that ATA reacts remarkably strongly with molecular oxygen. Additionally, the electrochemical reduction of ATA in the presence of a negative potential from -2.0 to $2.5 \mathrm{~V}$ generates a current signal, which is observed for hydrogen peroxide. Here we showed that ATA indicates a unique mechanism of YopH inactivation due to a redox process. We proposed that the potent inhibitory properties of ATA are a result of its strong binding in the YopH active site and in situ generation of hydrogen peroxide near catalytic cysteine residue.

\section{INTRODUCTION}

Yersinia genius contains three species of bacteria pathogenic to humans: plague-causing Yersinia pestis, septicemia-inducing Yesinia tuberculosis and Yersinia enterocolitica, which is responsible for a range of gastrointestinal disorders [1]. Yersinia pestis is transmitted by fleas while $Y$. tuberculosis and $Y$. enterocolitica are transmitted by the fecal oral route [2].

Yersinia $s p$. utilizes a type III secretion system for translocation of virulence effectors into the host cell [3]. All three Yersinia species contain a $70 \mathrm{~kb}$ plasmid that encodes a type III complex system and effectors (Yops).
During infection, Yersinia translocates Yops virulence effectors into a host cell leading to inhibition of the innate immune response [4].

One of Yersinia's outer membrane protein effectors is a highly active YopH protein tyrosine phosphatase, which is essential for virulence since the YopH mutant plasmid is avirulent [5]. YopH is causing deregulation of cellular functions, disrupting focal complex structures and blocking phagocytosis [6]. YopH disturbs the focal adhesions by dephosphorylation of the focal adhesion kinase (FAK) and suppresses the production of reactive oxygen species by macrophages [7]. 
The catalytic center of the YopH contains an amino acid sequence similar to eukaryotic protein tyrosine phosphatases (PTPs). There is a cysteine residue located at the bottom of the active site, which is essential for catalysis and enzymatic activity [8]. As for the eukaryotic PTP family, the catalytic cysteine is highly vulnerable to oxidation, which results in inactivation of the enzyme and depends on the oxidation state leads to formation in the active site, respectively, of an irreversible sulfenic acid residue or irreversible sulfinic and sulfonic acid residues [9].

Yersinia pestis still causes several thousand human cases per year and the climate change is increasing the risk of plague outbreaks in new geographic areas as well as spreading of its natural reservoirs [10]. For historical reasons and due to the fact that Yersinia pestis is one of the most virulent infectious agents threatening humans, there is an ever increasing risk of the use of $Y$. pestis by unauthorized groups as a biological weapon of terror [11]. The growing resistance of humans to antibiotics is one of the reasons to search for new treatment options and the bacterial virulence factor YopH is well positioned to become a new candidate for drug discovery [12].

There are libraries of chemical compounds that have been tested as YopH inhibitors and numerous of them were reported to inhibit YopH activity, mostly in micromolar concentrations [13]. Examples of such compounds include salicylic acid derivatives [14], natural substrate (pTyr) mimetics with carboxyl groups [15] or natural compounds, such as bromotyrosine alkaloids purified from a marine sponge [16].
One of the most effective YopH inhibitors is aurintricarboxylic acid (Figure 1A), with an $\mathrm{IC}_{50}$ value around $10 \mathrm{nM}$, which was evaluated by the Liang group [5] and confirmed by our results (Figure 1B). Aurintricarboxylic acid (ATA), a polyaromatic carboxylic acid derivative exhibiting polyanionic properties (Figure 1A), is a red dye and is not only known to inhibit protein tyrosine phosphatases but also nucleic acid binding enzymes, such as reverse transcriptase, DNA and RNA polymerase, topoisomerase and nuclease [17]. It is considered to be a potential anti-AIDS compound by preventing binding of HIV coat protein, gp120, to its CD4 receptor [17]. It has been demonstrated that ATA can inhibit the replication of viruses from several different families, such as the human immunodeficiency virus, also coronavirus, vesicular stomatitis virus and vaccinia virus. It does so by the inhibition of phosphatase activity of viral enzyme [18]. Because of its binding with cellular endonucleases, topoisomerases and various important signaling pathways, ATA has also been found to prevent apoptosis in a variety of cell models [19].

Taking the above studies into consideration, it is readily concluded that ATA is a remarkable compound, which should be seriously considered as a drug for infectious diseases, including epidemics caused by Yersinia $s p$.

We decided to evaluate the effectiveness of ATA as YopH inhibitor and to study possible mechanisms of ATA induced inactivation, as well as electrochemical properties of ATA. We performed computational docking and molecular dynamic studies to gain a molecular-level insight into the binding affinities and conformations of ATA in the YopH active site.<smiles>O=C(O)C1=CC(=C(c2ccc(O)c(C(=O)O)c2)c2ccc(O)c(C(=O)O)c2)C=CC1=O</smiles>

\begin{tabular}{lcc}
\hline \multicolumn{1}{c}{ PTPs } & $\mathbf{I C}_{50}$ value of ATA & $\begin{array}{c}\mathbf{I C}_{50} \text { value of } \\
\text { peroctanoic acid }\end{array}$ \\
\hline YopH Y.pestis & $10 \mathrm{nM}$ & $41 \mathrm{nM}$ \\
YopH & $100 \mathrm{nM}$ & $39 \mathrm{nM}$ \\
Y.entorocolitica & & \\
CD45 & $200 \mathrm{nM}$ & $25 \mathrm{nM}$ \\
\hline
\end{tabular}

\section{Aurintricarboxylic acid (ATA) $\mathrm{C}_{22} \mathrm{H}_{14} \mathrm{O}_{9}$}

Figure 1: ATA as YopHs inhibitor. A. The structure of aurintricarboxylic acid (ATA). B. $\mathrm{IC}_{50}$ values of ATA and peroctanoic acid for YopHs and CD45 inhibition. $\mathrm{IC}_{50}$ values were determined from a plot presenting ATA or peroctanoic acid concentration versus percentage of the enzymatic activity measured as absorbance with $p$ NPP substrate of recombinant CD45, YopH after 15 minutes incubation with inhibitors. 


\section{RESULTS}

\section{Inhibitory effects of ATA on YopH and CD45 enzymatic activity}

YopH recombinant phosphatase from Yersinia pestis and Yersinia eneterocolitica was treated with ATA and $\mathrm{IC}_{50}$ values were calculated. We also treated YopHs with peroctanoic acid, containing a peroxycarboxyl group with a higher oxidizing potency than that of a carboxyl group, studied by our group as a strong PTP inhibitor [20], in order to compare the inhibitory effects. In addition, the human CD45 recombinant protein tyrosine phosphatase was utilized to study the inhibitory properties of ATA on human PTP.

We found that ATA inactivates YopH and CD45 phosphatases at nanomolar concentrations. Interestingly, considering $\mathrm{IC}_{50}$ values, ATA was more effective against YopH from $Y$. pestis and YopH from $Y$. enterocolitica than against CD45 phosphatase (Figure 1B). Comparing the inhibitory effect of ATA with peroctanoic acid, ATA inactivated $Y$. pestis $\mathrm{YopH}$ with greater potency than peroctanoic acid, but peroctanoic acid was found to be a stronger inhibitor of CD45 (Figure 1B).

\section{The mechanism of ATA induced inactivation of YopH}

We prepared a reduction assay with dithiothreitol (DTT) to investigate the reversibility of ATA induced inhibition. We observed that ATA inactivates YopH reversibly and the ATA induced inhibition of YopH was completely reversed after a 20 minutes incubation process with DTT (Figure 2A).

Due to the fact that YopH, as other PTPs, contains an oxidation-sensitive cysteine located in the active site, we decided to prepare an NBD-Cl assay to calculate the amount of thiol groups after treatment with ATA. We tested the amount of thiol groups modified by NBD$\mathrm{Cl}$ forming Cys-S-NBD adducts. After treatment of recombinant YopH with $100 \mathrm{nM}$ ATA, the quantity of Cys-S-NBD adducts was significantly decreased in comparison with control (Figure 2B). Application of ATA has resulted in an over $50 \%$ reduction of thiolNBD adducts compared to control, suggesting that thiol groups in YopH after treatment with ATA are likely to be in oxidized form. Interestingly, the addition of catalase completely prevented ATA induced decrease of thiol adducts (Figure 2B).

We also observed that catalase not only prevents a decrease of thiol adducts but also prevents an inhibition of enzyme activity. The catalase pretreatment almost completely protected from ATA induced YopH inactivation (Figure 3A), as well as CD45 inactivation (Figure 3B). The elimination of inhibitory effects of ATA in the presence of catalase and a loss of thiol adducts after ATA treatment may lead to the conclusion that ATA is probably inactivating PTPs due to an oxidative mechanism.
A

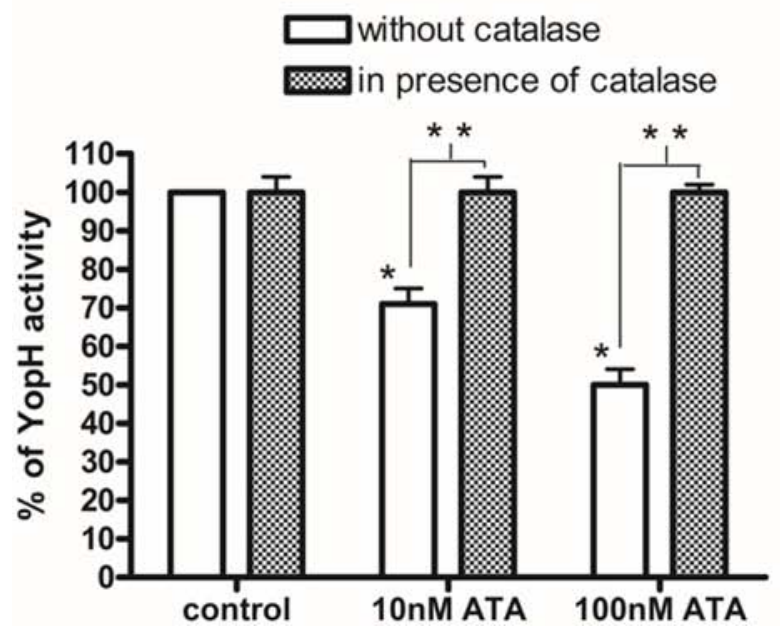

B

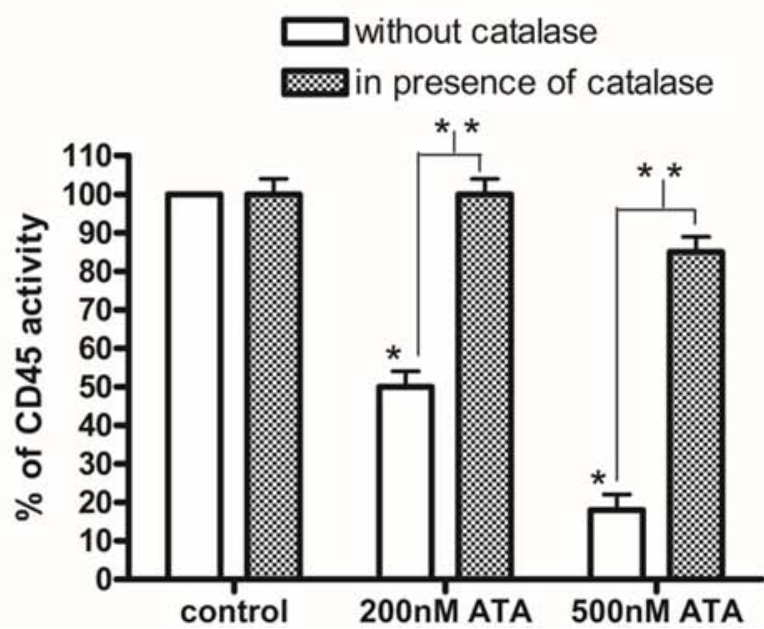

Figure 2: The reversibility and mechanism of ATA inhibition. A. Reduction assay of YopH Y. enterocolitica activity with DTT. Recombinant YopH was pretreated for 15 minutes with $100 \mathrm{nM}$ ATA and subsequently incubated with 10 mM DTT to reverse the ATA inhibition. The increase of activity of YopH was measured every minute on microplate reader as absorbance at $405 \mathrm{~nm}$ using $p$ NPP substrate. Data presented as percent of control. B. The amount of modified YopH thiol adducts with NBD (Cys-S-NBD adducts) after 15 minutes of treatment with $100 \mathrm{nM}$ ATA with/without catalase $(500 \mathrm{U} / \mathrm{ml})$. Data presented as absorbance $(420 \mathrm{~nm}), \mathrm{means} \pm \mathrm{SD}(n=3)$. One-way Anova test. * significantly different $(P<0.001)$. 
A

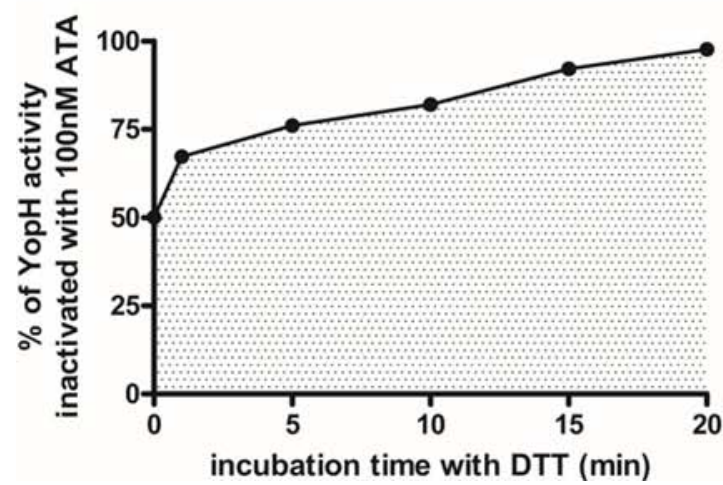

B

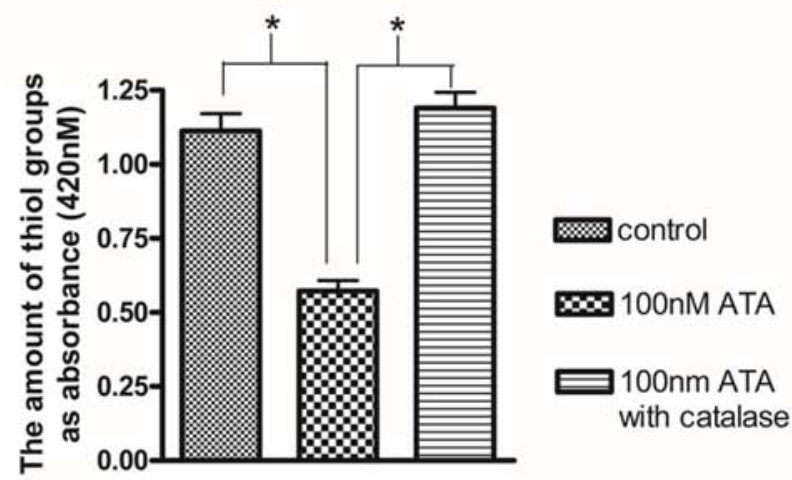

Figure 3: The elimination of ATA inhibitory properties by catalase. A. Activity of YopH after treatment with $10 \mathrm{nM}$ and $100 \mathrm{nM}$ ATA in presence and absence of catalase $(500 \mathrm{U} / \mathrm{ml})$. The enzymatic activity of YopH was measured on microplate reader as absorbance at $405 \mathrm{~nm}$ using $p$ NPP substrate. Data presented as percent of control, means $\pm \mathrm{SD}(n=10) . T$-test analysis of variance. * significantly different $(P<0.001)$ from control, **significantly different $(P<0.001)$ in pairs. B. Activity of CD45 after treatment with $200 \mathrm{nM}$ and $500 \mathrm{nM}$ ATA in presence and absence of catalase. The enzymatic activity of CD45 was measured on microplate reader as absorbance at $405 \mathrm{~nm}$ using $p$ NPP substrate. Data presented as percent of control, means $\pm \mathrm{SD}(n=10) . T$-test analysis of variance. * significantly different $(P<0.001)$ from control, ** significantly different $(P<0.001)$ in pairs.

\section{Electrochemical experiments}

We performed electrochemical measurement in order to investigate the oxidizing potency of ATA. In this study the signals from the redox process of ATA preformed in the DMSO solution were not observed (Figure 4A, grey line) in the region of oxidation-reduction potentials of oxygen (red line). In the solution of oxygen $\left(2.2 \cdot 10^{-4}\right.$ M) the presence of ATA causes significant changes in the reduction and oxidation process. When the concentration of ATA increased from $5.0 \cdot 10^{-5}$ to $3.7 \cdot 10^{-4} \mathrm{M}$ (dotted lines) the new reduction peak has appeared and the reoxidation process for oxygen disappeared.

A small addition of ATA caused significant changes in the cyclic voltammetric curve resulting in the appearance of a new cathodic peak at potential values form $0.74 \mathrm{~V}$ to $0.82 \mathrm{~V}$ (Figure $4 \mathrm{~A}$ ) (dotted lines). The disappearance of the anodic peak indicates that oxygen radicals react with the molecule of ATA. At the same time the appearance of a new peak is observed (blue line). This peak reached a constant value at $3.7 \cdot 10^{-4} \mathrm{M}$ of ATA concentration and is higher than the current peak for oxygen reduction (red line).

The analysis of cyclic voltammograms $(\mathrm{CVs})$ in a wide potential range (Figure 4B) indicates electrochemical reduction of ATA in the presence of negative potentials ranging from $-2.0 \mathrm{~V}$ to $-2.5 \mathrm{~V}$. The current signal obtained for ATA is very similar to the cyclic voltammograms observed for hydrogen peroxide. The value of the current obtained for ATA at a concentration of $4.9 \cdot 10^{-4} \mathrm{M}$ measured in DMSO solution is comparable to the hydrogen peroxide at a concentration of $1.5 \cdot 10^{-3} \mathrm{M}$ performed for potentials from $1.25 \mathrm{~V}$ to $2.5 \mathrm{~V}$. This may indicate that the hydrogen peroxide could be present during the redox process of ATA.

Comparison of reduction processes of hydrogen peroxide in an increasing range of concentrations from $1.6 \cdot 10^{-4} \mathrm{M}$ (black dotted line) to $6.0 \cdot 10^{-3} \mathrm{M}$ (blue line) (Figure 4C) measured in the ranges from $-1.0 \mathrm{~V}$ to $-2.5 \mathrm{~V}$ suggests that during the redox process of ATA the hydrogen peroxide could appear at very low concentrations.

\section{Docking studies}

ATA and natural substrate phosphotyrosine (pTyr) molecules were docked into the 3D structure of YopH in order to investigate the possible binding conformation and affinity. We performed blind flexible docking and retained top 30 conformations from docking runs. In all 30 conformations pTyr and ATA are bound to the active site of YopH, as shown in Figure 5A for phosphotyrosine and Figure 5B for ATA. The docking studies showed that ATA can be easily accommodated inside the binding site and binds specifically in a catalytic center of YopH, in a similar manner to that of the natural substrate, phosphotyrosine (Figure 5A, 5B).

\section{The molecular dynamics simulations of YopH with ATA}

To study the binding conformation of ATA in the YopH active site we performed molecular dynamics simulations using Amber12 and identified top scoring 
A

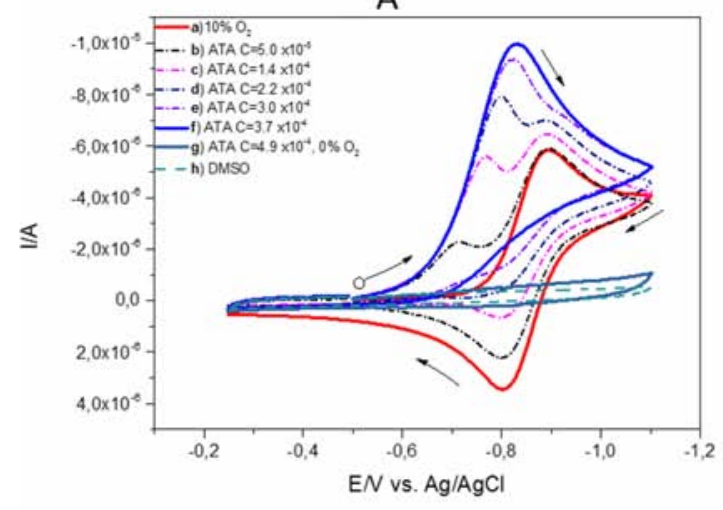

B

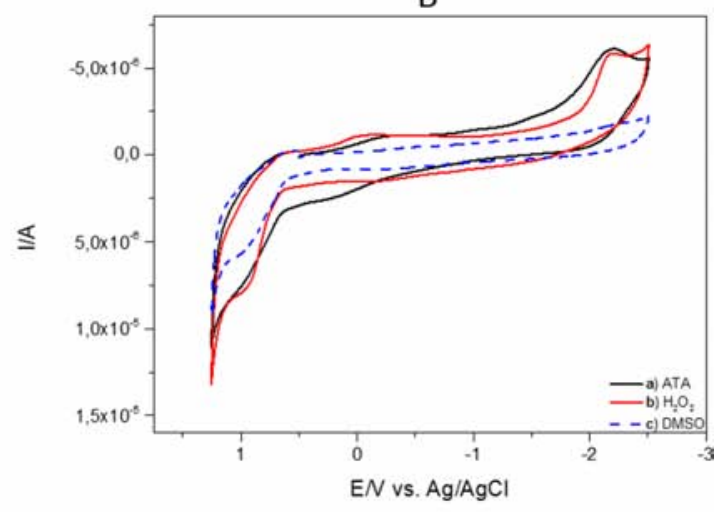

C

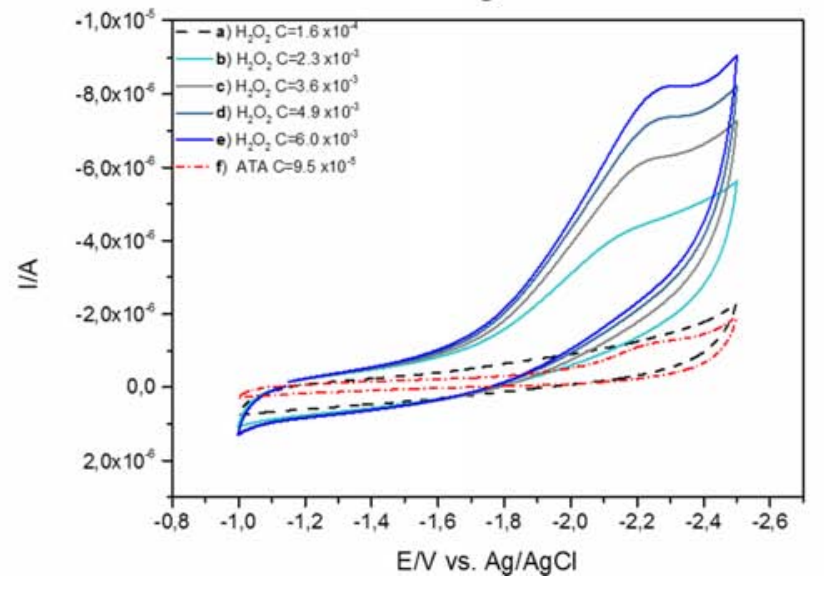

Figure 4: Cyclic voltammetry of ATA. A. A series of cyclic voltammograms (CVs): a) of $2.2 \cdot 10^{-4} \mathrm{M}$ of oxygen in DMSO solutions (red line) at gradually increasing concentration of ATA: b) $5.0 \cdot 10^{-5} \mathrm{M}$, c) $1.4 \cdot 10^{-4} \mathrm{M}$, d) $2.2 \cdot 10^{-4} \mathrm{M}$, e) $3.0 \cdot 10^{-4} \mathrm{M}$, (dotted lines), f) $3.7 \cdot 10^{-4} \mathrm{M}$ (blue line), g) $4.9 \cdot 10^{-4} \mathrm{M}$ of ATA without oxygen (grey line) and h) DMSO in $0.1 \mathrm{M}$ TBAP, conditions: scan rate $100 \mathrm{mV} / \mathrm{s}$. B. Cyclic voltammograms (CVs) of a) ATA - concentration $4.9 \cdot 10^{-4} \mathrm{M}$ in DMSO solution (black line) b) hydrogen peroxide - concentration $1,5 \cdot 10^{-3} \mathrm{M}$ (red line) c) potential window obtained in DMSO in $0.1 \mathrm{M}$ TBAP solution (blue dotted line), conditions: scan rate $100 \mathrm{mV} / \mathrm{s}$. C. Comparison of cyclic voltammograms (CVs) of a - e) hydrogen peroxide at different concentrations from $1.6 \cdot 10^{-4} \mathrm{M}$ (black dotted line) to $6.0 \cdot 10^{-3} \mathrm{M}$ (blue line) and ATA at concentration of $9.5 \cdot 10^{-5} \mathrm{M}$, (red dotted line), conditions: scan rate $100 \mathrm{mV} / \mathrm{s}$.

poses from docking studies. The interactions of ATA in the YopH binding site are presented as a PLIF diagram (protein ligand interaction fingerprints) in Figure 6A. The dotted line around the molecule shows solvent contact and dotted arrows represent hydrogen bonds between amino acid residues from YopH and ATA. In the predicted binding pose two carboxyl groups of ATA are directed toward essential Cys403 and Arg409 residues in the YopH active site. Under such steric conditions there is likelihood of hydrogen bond formation between the arginine residue and carboxyl groups of ATA. As shown in the PLIF diagram, ATA has been able to utilize its polar groups to interact electrostatically with Cys403, Arg409 and water (Figure 6A). The positively charged arginine residue of the YopH active site is likely to attract the negatively charged carboxyl groups from ATA.

\section{The binding energies of ATA and pTyr to YopH}

To indicate the binding affinities of ATA and pTyr to YopH we calculated the binding free energy for the complex. Figures $6 \mathrm{~B}$ and $6 \mathrm{C}$ show the binding energies to YopH and their components for ATA and pTyr respectively. The components are described below:

$$
\begin{aligned}
\Delta E_{v d w}= & \text { the Van der Waals contribution from MM. } \\
\Delta E_{\text {ele }}= & \text { Electrostatic energy as calculated by the } \\
& \text { MM force field } \\
\Delta G_{\text {polar }}= & \text { The electrostatic contribution to the } \\
& \text { solvation free energy calculated by } \mathrm{PB} \text { or } \\
& \text { GB respectively } \\
\Delta G_{\text {non-polar }}= & \begin{array}{l}
\text { Nonpolar contribution to the solvation free } \\
\text { energy calculated by an empirical model }
\end{array} \\
\Delta G_{\text {bind }}= & \text { final estimated binding free energy } \\
& \text { calculated from the terms above }\left(\mathrm{kcal} / \mathrm{mol}^{-1}\right.
\end{aligned}
$$




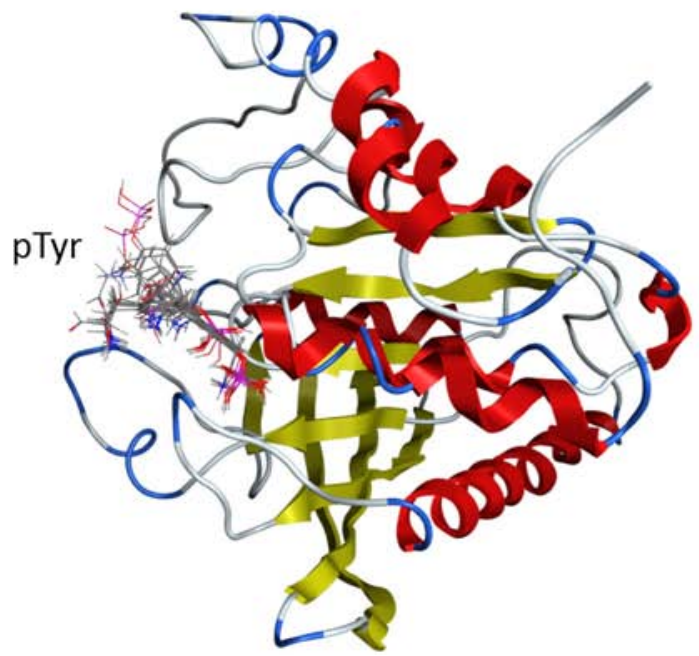

B

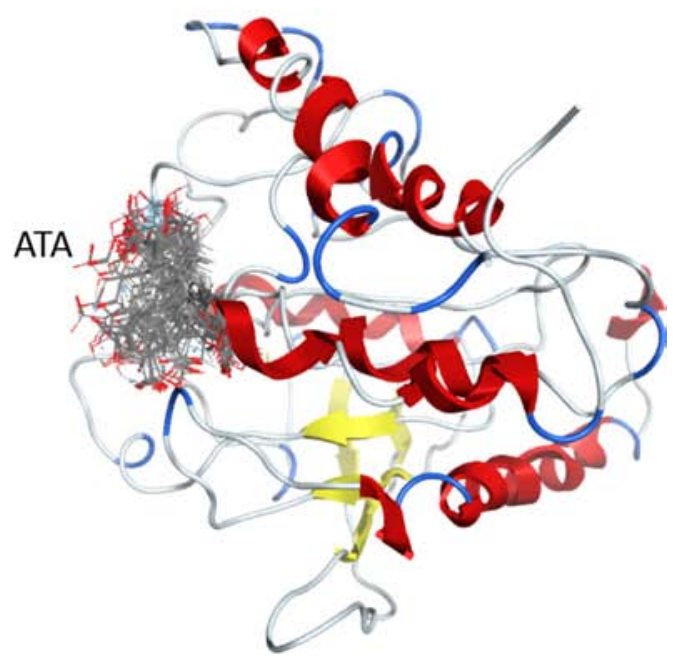

Figure 5: The blind flexible docking of pTyr and ATA molecules into the YopH 3D structure. A. The site of binding for top 30 conformations of pTyr obtained from docking of pTyr into the YopH structure. In each conformation pTyr binds in the YopH active site. B. The site of binding for top 30 conformations of ATA obtained from docking of ATA into the YopH structure. In each conformation ATA binds in the YopH active site.

A

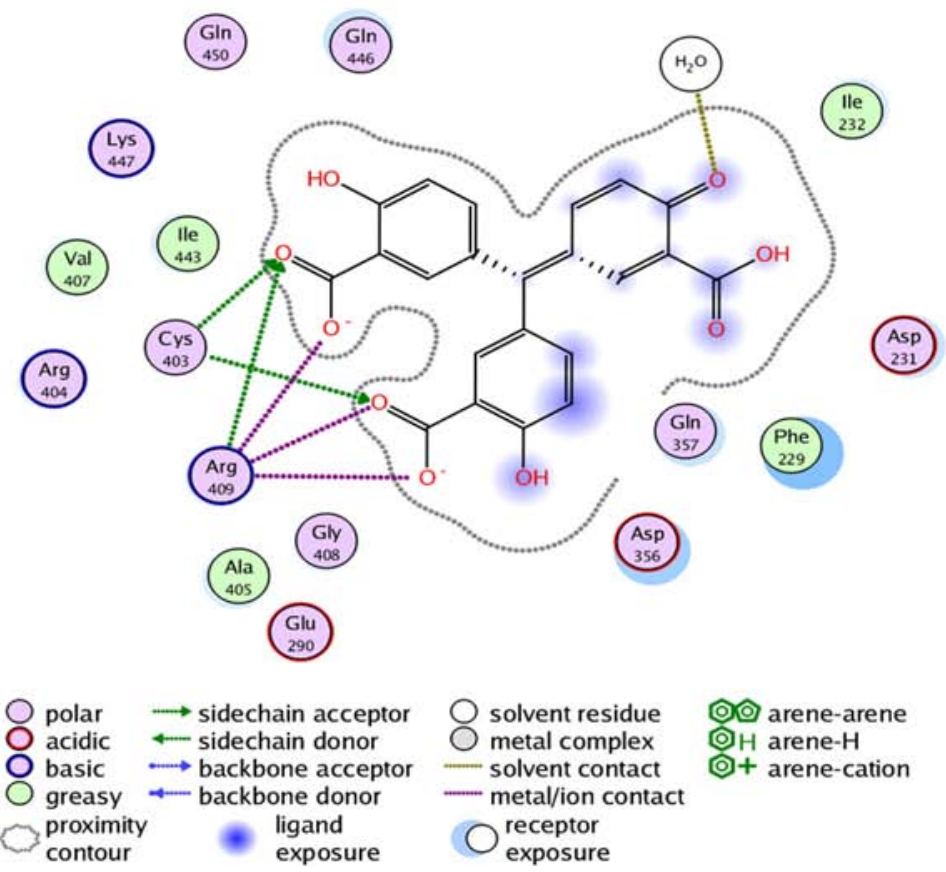

B

\begin{tabular}{|c|l|l|}
\hline $\begin{array}{l}\text { YopH-ATA binding energy } \\
\text { components }\end{array}$ & $\begin{array}{l}\text { Contribution } \\
\left(\mathrm{kcal} / \mathrm{mol}^{-1}\right)\end{array}$ & SD \\
\hline$\Delta E_{\text {vdw }}$ & -32.07 & \pm 3.25 \\
\hline$\Delta E_{\text {ele }}$ & -344.03 & \pm 16.47 \\
\hline$\Delta G_{\text {polar }}$ & 349.18 & \pm 14.29 \\
\hline$\Delta G_{\text {non-polar }}$ & -4.68 & \pm 0.15 \\
\hline$\Delta G_{\text {bind }}$ & -31.60 & \pm 5.49 \\
\hline
\end{tabular}

$\mathrm{C}$

\begin{tabular}{|c|l|l|}
\hline $\begin{array}{c}\text { YopH-pTyr binding energy } \\
\text { components }\end{array}$ & $\begin{array}{l}\text { Contribution } \\
\left(\mathrm{kcal}_{\mathrm{mol}} \text { ) }\right.\end{array}$ & SD \\
\hline$\Delta E_{\text {vdw }}$ & -35.70 & \pm 2.27 \\
\hline$\Delta E_{\text {ele }}$ & -47.74 & \pm 5.83 \\
\hline$\Delta G_{\text {polar }}$ & 64.69 & \pm 4.82 \\
\hline$\Delta G_{\text {non-polar }}$ & -4.87 & \pm 0.07 \\
\hline$\Delta G_{\text {bind }}$ & -23.63 & \pm 4.37 \\
\hline
\end{tabular}

Figure 6: Molecular dynamic simulation of ATA in the YopH active site and binding energies for ATA and pTyr to YopH. A. The PLIF diagram for the best binding pose of ATA in the YopH binding site. In predicted binding pose, two carboxyl groups of ATA are directed toward essential Cys403 and Arg409 residues in the active site. There are electrostatic interactions between polar groups of ATA with Cys403, Arg409 and water. B. Binding free energy and its components for the YopH-ATA complex by MM/GBSA methods $\left(\mathrm{Kcal} \mathrm{mol}^{-1}\right)$. C. Binding free energy and its components for the YopH-pTyr complex by MM/GBSA methods $\left(\mathrm{kcal}^{\mathrm{mol}}{ }^{-1}\right)$. 
The final estimated binding energy was calculated by the following equations:

$$
\begin{aligned}
& \Delta G_{\text {bind }}=G_{\text {Complex }}=G_{\text {Receptor }}=G_{\text {Ligand }} \\
& \text { Where } \mathrm{G} \text { stands for free energy. } \\
& \Delta G_{\text {bind }}=\Delta E_{M M} \pm \Delta G_{\text {Polar }} \pm \Delta G_{\text {non-Polar }} \\
& \Delta G_{\text {Polar }} \text { is the polar contribution to the solvation }
\end{aligned}
$$
free energy and $\Delta G_{\text {non-Polar }}$ is the non-polar contribution to the solvation free energy and is defined as $\Delta G_{n o n-P o l a r}=\gamma S A S A \pm b$, where $\gamma=0.0072 \mathrm{Kcal} \mathrm{mol}^{-1}$ $\AA^{-1}$ and $b=0$ for AMBER-based GBSA calculations. SASA stands for the solvent accessible surface area. $T \Delta S$ represents the entropic term and needs to be calculated by normal mode analysis, which is computationally expensive and since other ligands will bind to the same protein in the same binding site, we neglected the entropic contribution to the binding free energy in our calculations.

$$
E_{M M}=E_{\text {int }} \pm E_{\text {ele }} \pm E_{v d w}
$$

$E_{M M}$ is the molecular mechanics contribution to binding in vacuo expressed as a sum of the internal, electrostatic and van der Waals' contributions. Since we use a single trajectory approach, internal energy $E_{\text {int }}$ will be approximately cancelled, thereby:

$$
E_{M M}=E_{\text {ele }} \neq E_{v d w}
$$

The binding affinity indicates that ATA exhibits stronger binding to $\mathrm{YopH}$ than its natural substrate phosphotyrosine (Figure 6B, 6C). The comparison between binding energies of ATA and pTyr into YopH shows a much stronger van der Waals interaction for the YopH-ATA as compared to the YopH-pTyr complex.

\section{DISCUSSION}

Earlier studies of YopH inhibitors revealed aurintricarboxylic acid as the strongest inhibitor of Yersinia YopH virulence effector [5]. In this study our objective was to evaluate the effectiveness and possible mechanisms of ATA induced inactivation of YopH. Our experiments with recombinant YopH phosphatase confirmed that ATA can effectively inactivate YopH phosphatases at nanomolar concentration.

Taking into consideration the fact that YopH has a similar amino-acid sequence in the active site as those for other PTPs [8], including human ones, we compared the effect of ATA on human CD45 phosphatase. Protein tyrosine phosphatase CD45 is abundantly expressed in all non-nucleated haematopoietic cells and is critical for T-cell and B-cell functions. It dephosphorylates the Src kinases, therefore is important to immune-receptor modulated signaling. The inactivation of CD45 would lead to serious complications for the host immunity [21]. We found that ATA is more effective against YopH phosphatase than CD45.

The reduction assay indicated that ATA is a reversible inhibitor of YopH and that the presence of catalase abolished the effect of ATA on YopH. The inactivation of the inhibitory activity of the compound in the presence of catalase is likely through an oxidative mechanism, due to the fact that catalase is an enzyme protecting cells from reactive oxygen species by decomposition of hydrogen peroxide [22]. Some of the PTP inhibitors are acting by production of hydrogen peroxide, which is suggested as a key regulator of PTP activity in cells [23]. ATA loses the inhibitory properties against PTPs in the presence of catalase, which shows that the mechanism of ATA induced inactivation of YopH is probably involved with the oxidation of catalytic cysteine in an active site. It is confirmed also by the loss of unoxidized thiol groups in YopH after treatment with ATA.

In aprotic solvents, such as dimethyl sulfoxide (DMSO) electrochemically generated superoxide anions $\left(\mathrm{O}_{2}^{-}\right)$are solvated far less efficiently than in water [24]. This provides an opportunity to study the interactions between the reactive oxygen species and organic compounds and also to examine if organic compounds react either with an inert form of oxygen or its reduced forms. The cyclic voltammetry for generation and detection of superoxide anions by the electrochemical reduction of molecular oxygen is very useful and easy to follow the reaction of superoxide anions with bioactive compounds at the electrode surface [25]. The obtained results indicate that ATA directly reacts with oxygen and reactive oxygen forms in DMSO solution. Additionally, the electrochemical reduction of ATA negative reduction potential $(-2.0$ to $2.5 \mathrm{~V})$ generates a current signal, which has been observed for hydrogen peroxide.

The docking studies showed that ATA bonds specifically in the active site of YopH. ATA can accommodate itself well inside the binding site as compared to pTyr and that may be the reason for a higher binding affinity of ATA to YopH and ATA as compared to pTyr. By performing molecular dynamics simulations of the complex we found that two carboxyl groups of ATA bind directly toward the essential amino-acid residues for enzyme catalysis in the active site. Cys403 and Arg409 are involved in the reaction of dephosphorylation of substrate on tyrosine residues. The binding conformation of ATA in the YopH active site contributed to the relatively strong calculated binding affinity of ATA in comparison to natural substrate.

In conclusion, we demonstrated that ATA effectively decreases the Yersinia YopH activity and has stronger binding to YopH active site than natural substrate. Here we proposed a unique mechanism of ATA caused inactivation of YopH involved with its redox process leading to generation of hydrogen peroxide. We suggest that the potent inhibitory properties of ATA are due to strong binding in the YopH active site and generation of hydrogen peroxide directly inside the active site. The studies on inhibitory properties of ATA against YopH are a good starting point for the synthesis of analogous inhibitors and potentially lead to clinical applications. Further studies on ATA analogs and cellular aspects of ATA are underway. 


\section{MATERIALS AND METHODS}

\section{Recombinant PTP YopH and CD45 activity assay}

Bacterial recombinant YopH protein tyrosine phosphatase from Yersinia pestis was obtained from Millipore and YopH from Yersinia enterocolitica was obtained from Calbiochem. Human recombinant CD45 was obtained from Sigma-Aldrich. The solutions of the recombinant PTPs were prepared in $10 \mathrm{mM}$ HEPES buffer $\mathrm{pH}$ 7.4. The final concentration of phosphatase in reaction samples was $0.8 \mu \mathrm{g} / \mathrm{mL}(10 \mathrm{nM})$. The YopHs and CD45 enzymes were untreated (control) or treated with solution of ATA and peroctanoic acid. The assay was performed in 96-well microplates, and the final volume of each sample was $200 \mu \mathrm{L}$. The enzymatic activities of YopHs and CD45 were measured using $1 \mathrm{mM}$ chromogenic substrate paranitrophenyl phosphate ( $p$ NPP) in 10 mM HEPES buffer $\mathrm{pH} 7.4$, at $37^{\circ} \mathrm{C}$. Phosphatase hydrolyzed $p$ NPP to paranitrophenol and inorganic phosphate. Para-nitrophenol is an intensely yellow colored soluble product under alkaline conditions. The increase in absorbance (due to para-nitrophenol formation) is linearly proportional to enzymatic activity concentration (with excessive substrate, i.e. zero-order kinetics) and was assessed at $405 \mathrm{~nm}$ on a microplate reader Jupiter (Biogenet) using DigiRead Communication Software (Asys Hitech GmbH).

\section{Reduction assay with DTT}

Subsequently, recombinant phosphatase YopH that had been previously inactivated by ATA, was then treated with $10 \mathrm{mM}$ dithiothreitol (DTT), and the samples were incubated at $37^{\circ} \mathrm{C}$ to reverse the inactivation, if possible. Restoration of YopH enzymatic activity was measured every minute as an increase of absorbance taken at $405 \mathrm{~nm}$ as previously described.

\section{YopH thiol adduct assay}

The recombinant phosphatase YopH was inactivated by ATA and the amount of modified YopH thiol adduct with NBD (Cys-S-NBD adduct) was measured after 30 minutes incubation with NDB-Cl $(0.6 \mathrm{mM}$ in a $0.5 \mathrm{~mL}$ sample) as absorbance at $420 \mathrm{~nm}$ with a spectrophotometer.

\section{Electrochemical analysis}

Cyclic voltammetry (CV) experiments were performed using an Autolab potentiostat/galvanostat (model PGSTAT30) supported by the GPES software. The experiments were performed at room temperature in DMSO solution with $0.1 \mathrm{M}$ tetrabutylammonium tetrafluoroborate (TBAP) as the supporting electrolyte, at a scan rate of $100 \mathrm{mV} / \mathrm{s}$ under argon atmosphere or in the oxygen-saturated DMSO solution. A conventional three-electrode configuration consisting of a glassy carbon electrode (GC), a working electrode with a 3-mm diameter. The platinum wire used as the counter electrode and an $\mathrm{Ag} / \mathrm{AgCl}$ in $3 \mathrm{M} \mathrm{KCl}$ reference electrode were used, respectively. Each time before use, the working $\mathrm{GC}$ was polished with $\mathrm{Al}_{2} \mathrm{O}_{3}$ powder on a wet pad, then the electrode was washed with nanopure water, and dried under a stream of nitrogen. The oxygen-saturated DMSO solution used to perform measurement was obtained by the procedure described in the literature [26]. The concentration of oxygen in oxygen-saturated DMSO at $298 \mathrm{~K}$ was $2.2 \cdot 10^{-3} \mathrm{M}$ [27]. During all the experiments with oxygen the air was carefully excluded from the measurement cell and the oxygen was bubbled directly into the cell in order to obtain desirable concentration. The error in oxygen concentration of all measurement is estimated to be approximately $1 \%$. The oxygen flow was adjusted by a mass flow controller (HiTec, Bronkhorst).

\section{Docking studies}

The initial structure of YopH was imported from the RCSB protein data bank (http://www.pdb.org) with code 2YDU.pdb [28]. The structure was minimized using taff.ff forcefield of the Molecular Operating Environment software (MOE, chemical computing group). Chain A of this pdb file contains 306 residues. The ligand was removed from this pdb file and aurintricarboxylic acid (ATA) and phosphotyrosine (pTyr) were docked into the structure of YopH. A blind flexible docking simulation was performed, where the binding site was assumed to be the entire protein. The side chains were kept free to move during forcefield refinement. Alpha PMI is the placement method used with default settings (sample per conformation $=10$, maximum poses $=250$ ). London dG rescoring was used with Alpha PMI placement. Termination criteria for forcefield refinement were set as gradient $=0.001$ and interactions $=500$.

\section{Molecular dynamics simulations}

Top scoring poses from docking that interacted with Cys403 were retained for molecular dynamics simulations using amber12. We allowed Leap module of Amber [29] to add missing hydrogen atoms and heavy atoms using the Amber force field (ff10) parameters [30]. To neutralize the charge of the system, we added sodium/chloride ions. The model was immersed in a truncated cubical shell of TIP3P water [31]. A time step of $2 \mathrm{fs}$ and a direct-space non-bonded cutoff of $10 \AA$ were used. After the protein preparation, all systems were minimized to remove the steric clashes that occurred. The systems were then gradually heated from 10 to $300 \mathrm{~K}$ over a period of $50 \mathrm{ps}$ and then maintained in the isothermal-isobaric ensemble (NPT) at a target temperature of $300 \mathrm{~K}$ and a target pressure of 1 bar using a Langevin thermostat $[32,33]$ and a Berendsen barostat with a collision frequency of 2 ps 
and a pressure relaxation time of $1 \mathrm{ps}$, respectively. We constrained hydrogen bonds using the SHAKE algorithm [34]. We have used the velocity-Verlet algorithm (default algorithm for the Amber MD package) for MD simulations. Particle mesh Ewald (PME) procedure was used to treat long-range electrostatic interactions using default parameters [35]. After bringing the systems at our suitable temperature and pressure of $300 \mathrm{~K}$ and 1 bar, respectively and equilibrating the system for $500 \mathrm{ps}$, the production run was continued for $20 \mathrm{~ns}$ in the isothermal-isobaric ensemble at the target temperature of $300 \mathrm{~K}$ and target pressure of 1 bar using the same Langevin thermostat and Berendsen barostat. The structures in the trajectories were collected at $10 \mathrm{ps}$ intervals. The analysis of trajectories was performed with the Ptraj module of Amber.

\section{Binding affinity calculations}

For the binding free energy calculations, we used the standard MM/GBSA method [36]. MMPBSA.py python script was used for MM/GBSA calculations [37]. Before the MM/GBSA analysis, all water molecules and the sodium ions were excluded from the trajectory. The dielectric constant used for the solute and surrounding solvent was 1 and 80, respectively. During the analysis of the MM/GBSA trajectory, snapshots were gathered at 10 ps intervals from the last 500 ps of the 20 ns trajectory.

\section{Statistical analysis}

The experiments were performed at least three times. The data were applied and analyzed with GraphPad Prism (GraphPad Software v.4). Statistical analyses were performed using ANOVA combined with Tukey's test or $\mathrm{T}$ test combined with Wilcoxon test. The data were expressed as means \pm SD. Differences between means were considered significant for $P<0.05$.

\section{ACKNOWLEDGMENTS}

JAT gratefully acknowledges support from Natural Sciences and Engineering Research Council of Canada (NSERC). Computations were performed using WestGrid Canada resources and the Pharmamatrix cluster. MW acknowledges to Medical University of Gdansk Funding No. ST46.

\section{FUNDING}

This work was supported by Grant No. 2012/07/N/ NZ1/00012 from Polish National Science Center. The analysis of iron chelator ATA on CD45 was performed with support from Medical University of Gdansk MN Funding No. 01-0172/08/259. The funders had no role in study design, data collection and analysis, decision to publish, or preparation of the manuscript.

\section{CONFLICTS OF INTEREST}

The authors declare no conflicts of interest.

\section{REFERENCES}

1. Trosky JE, Liverman AD, Orth K. Yersinia outer proteins: Yops. Cell Microbiol. 2008; 10:557-65.

2. Achtman M, Morelli G, Zhu P, Wirth T, Diehl I, Kusecek B, Vogler AJ, Wagner DM, Allender CJ, Easterday WR, Chenal-Francisque V, Worsham P, Thomson NR, et al. Microevolution and history of the plague bacillus, Yersinia pestis. Proc Natl Acad Sci USA. 2004; 101:17837-17842.

3. Bahta M, Burke TR. Yersinia pestis and approaches to targeting its outer protein $\mathrm{H}$ protein-tyrosine phosphatase (YopH). Curr Med Chem. 2012; 19:5726-34.

4. Viboud GI, So SS, Ryndak MB, Bliska JB. Proinflammatory signalling stimulated by the type III translocation factor YopB is counteracted by multiple effectors in epithelial cells infected with Yersinia pseudotuberculosis. Mol Microbiol. 2003; 47:1305-15.

5. Liang F, Huang Z, Lee SY, Liang J, Ivanov MI, Alonso A, Bliska JB, Lawrence DS, Mustelin T, Zhang ZY. Aurintricarboxylic acid blocks in vitro and in vivo activity of YopH, an essential virulent factor of Yersinia pestis, the agent of plague. J Biol Chem. 2003; 278:41734-41.

6. Deleuil F, Mogemark L, Francis MS, Wolf-Watz H, Fällman M. Interaction between the Yersinia protein tyrosine phosphatase $\mathrm{YopH}$ and eukaryotic Cas/Fyb is an important virulence mechanism. Cell Microbiol. 2003; 5:53-64.

7. Trulzsch K, Sporleder T, Leibiger R, Russmann $H$, Heesemann J. Yersinia as oral live cerrier vaccine: Influence of Yersinia outer proteins (Yops) on the T-cell response. Int J Med Microbiol. 2008; 298:59-67.

8. Black DB, Marie-Cardine A, Schraven B, Bliska JB. The Yersinia tyrosine phosphatase YopH targets a novel adhesion-regulated signalling complex in macrophages. Cell Microbiol. 2000; 2:401-414.

9. Ostman A, Frijhoff J, Sandin A, Bohmer F. Regulation of protein tyrosine phosphatases by reversible oxidation. J Biochem. 2011; 150:345-356.

10. Stenseth NC, Atshabar BB, Begon M, Belmain SR, Bertherat E, Carniel E, Gage KL, Leirs H, Rahalison L. Plague: past, present, and future. PLoS Med. 2008; 5:9-13.

11. Hawley RJ, Eitzen EM. Biological weapons - a primer for microbiologists. Annu Rev Microbiol. 2001; 55:235-53.

12. Bohmer F, Szedlacsek S, Tabernero L, Ostman A, den Hertog J. Protein tyrosine phosphatases structure-function relationships in regulation and pathogenesis. FEBS J. 2012; 280:413-431.

13. Heneberg P. Finding the smoking gun: protein tyrosine phosphatases as tools and targets of unicellular microorganisms and viruses. Curr Med Chem. 2012; 19:1530-66. 
14. Huang Z, He Y, Zhang X, Gunawan A, Wu L, Zhang ZY, Wong CF. Derivatives of salicylic acid as inhibitors of YopH in Yersinia pestis. Chem Biol Drug Des. 2010; 76:85-99.

15. Zhang Z-Y. Inhibitors of Protein Tyrosine Phosphatases. In Hanbook of Cell Signaling (Dennis, E.A.). Academic PressNew York: 2003; :677-684.

16. Yin S, Davis RA, Shelper T, Sykes ML, Avery VM, Elofsson M, Sundin C, Quinn RJ. Pseudoceramines A-D, new antibacterial bromotyrosine alkaloids from the marine sponge Pseudoceratina sp. Org Biomol Chem. 2011; 9:6755-60.

17. Bardhan M, Chowdhurry J, Ganguly T. Investigations on the interactions of aurintricarboxylic acid with bovine serum albumin: Steady state/time resolved spectroscopic and docking studies. J Photochem Photobiol B. 2011; 102:11-19.

18. Myskiw C, Deschambault Y, Jefferies K, He H, Cao J. Aurintricarboxylic acid inhibits the early stage of Vaccinia virus replication by targeting both cellular and viral factors. J Virol. 2007; 81:3027-3032.

19. Tsi CJ, Chao Y, Chen CW, Lin WW. Aurintricarboxylic acid protects against cell death caused by lipopolysaccharide in macrophages by decreasing inducible nitric-oxide synthase induction via I $\mathrm{B}$ mitogen-activated protein kinase inhibition. Mol Pharmacol. 2002; 101:90-101.

20. Kuban-Jankowska A, Gorska M, Tuszynski JA, Churchill CDM, Winter P, Klobukowski M, Wozniak M. Inactivation of protein tyrosine phosphatases by peracids correlates with the hydrocarbon chain length. Cell Physiol Biochem. 2015. 36:1069-1083.

21. Hermiston ML, Zikherman J, Zhu JW. CD45, CD148, and Lyp/Pep: critical phosphatases regulating Src family kinase signaling network in immune cells. Immunol Rev. 2009; 228:288-311.

22. Chelikani P, Fita I, Loewen P. Diversity of structures and properties among catalases. Cell Mol Life Sci. 2004; 61:192-208.

23. Bova MP, Mattson MN, Vasile S, Tam D, Holsinger L, Bremer M, Hui T, McMahon G, Rice A, Fukuto JM. The oxidative mechanism of action of ortho-quinone inhibitors of protein-tyrosine phosphatase alpha is mediated by hydrogen peroxide. Arch Biochem Biophys. 2004; 429:30-41.

24. Jeziorek D, Ossowski T, Liwo A, Dyl D, Nowacka M, Woznicki W. Theoretical and electrochemical study of the mechanism of anthraquinone-mediated one-electron reduction of oxygen: the involvement of adducts of dioxygen species to anthraquinones. J Chem Soc Perkin Trans. 1997; 2:229-236.
25. Ortiz ME, Nunez-Vergara LJ, Squella JA. Cyclic, voltammetric behaviour of the $\mathrm{O} 2 / \mathrm{O} 2$ - redox couple at a HMDE and its interaction with nisoldipine. J Electroanal Chem. 2002; 519:46-52.

26. Ossowski T, Pipka P, Liwo A, Jeziorek D. Electrochemical and UV-spectrophotometric study of oxygen and superoxide anion radical interaction with anthraquinone derivatives and their radical anions. Electrochimica Acta. 2000; 45:3581-3587.

27. Audri RL, Allen AO, Bielski BH. Some observations on the chemistry of KO2-DMSO solutions. FEBS Lett. 1981; 35:265-267.

28. Kim SE, Bahta M, Lountos GT, Ulrich RG, Burke TR Jr, Waugh DS. Isothiazolidinone (IZD) as a phosphoryl mimetic in inhibitors of the Yersinia pestis protein tyrosine phosphatase YopH. Acta Crystallogr D Biol Crystallogr. 2011; 67:639-45.

29. Case DA, Cheatham TE, Darden T, Gohlke H, Luo R, Merz KM, Onufriev A, Simmerling C, Wang B, Woods RJ. The Amber biomolecular simulation programs. J Comput Chem. 2005; 26:1668-88.

30. Lindorff-Larsen K, Piana S, Palmo K, Maragakis P, Klepeis JL, Dror RO, Shaw DE. Improved side-chain torsion potentials for the Amber ff99SB protein force field. Proteins. 2010; 78:1950-8.

31. Jorgensen WL, Chandrasekhar J, Madura JD, Impey RW, Klein ML. Comparison of simple potential functions for simulating liquid water. J Chem Phys. 1983; 79:926-935.

32. Izaguirre JAC, Catarello DP, Wozniak JM, Skeel RD. Langevin stabilization of molecular dynamics. J Chem Phys. 2001; 114:2090-2098.

33. Berendsen HJC, Postma JPM, van Gunsteren WF, DiNola A, Haak JR. Molecular dynamics with coupling to an external bath. J Chem Phys. 1984; 81:3684-3690.

34. Ryckaert JPC, Ciccotti G, Berendsen HJC. Numerical integration of the cartesian equations of motion of a system with constraints: Molecular dynamics of n-alkanes. J Comput Phys. 1977; 23:327-341.

35. Darden T, York D, Pedersen L. Particle mesh Ewald-an $\mathrm{N}-\log (\mathrm{N})$ method for Ewald sums in large systems. J Chem Phys. 1993; 98:10089-10092.

36. Gohlke H, Case DA. Converging free energy estimates: MM-PB(GB)SA studies on the protein-protein complex Ras-Raf. J Comput Chem. 2004; 25:238-50.

37. Miller BR, McGee TD, Swails JM, Homeyer N, Gohlke H, Roitberg AE. MMPBSA.py: An Efficient Program for EndState Free Energy Calculations. J Chem Theory Comput. 2012; 8:3314-3321. 